\title{
Functionally graded NiTi alloy with exceptional strain-hardening effect fabricated by SLM method
}

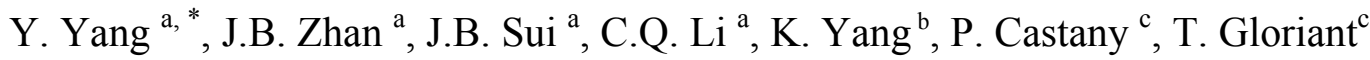 \\ ${ }^{a}$ School of Electromechanical Engineering, Guangdong University of Technology, Guangzhou \\ 510006, China \\ ${ }^{\mathrm{b}}$ Institute of Metal Research, Chinese Academy of Sciences, Shenyang 110016, China \\ ${ }^{c}$ Univ Rennes, INSA Rennes, CNRS, ISCR UMR 6226, 35000, Rennes, France \\ Corresponding Author: yy@alum.imr.ac.cn
}

\begin{abstract}
:
In this study, we have employed a repetitive laser scanning strategy to fabricate a three-dimensional functionally graded NiTi alloy by SLM method. The microstructure shows gradient characterized by the increase of volume fraction for B19' phase. An exceptional strain-hardening associated with continuous increase of mechanical recoverable strain is obtained in such material. The gradient of functionality is attributed to the superimposition of multi-deformation mechanisms originating from the microstructural gradient. The deformation mechanisms are dominated by reorientation of B19' variants at early stage of deformation and then by stress-induced martensitic transformation at higher level of stress.
\end{abstract}

Keywords: SLM, NiTi alloys, functionally graded, phase transformation

The NiTi alloy is the most widely used metallic shape memory alloy due to its superior shape memory effect and superelasticity. The maximum recoverable strain is proved to be up to $8 \%$ [1]. Other features including low Young's modulus, high damping capacity, good biocompatibility and corrosion resistance all enable the NiTi alloy an excellent candidate material for actuators in both aerospace and bio-engineering areas [2, 3]. As the origin of these unique properties, the reversible thermo-elastic martensitic transformation between the B2 phase and the B19' phase has been investigated [4]. However, the intrinsic narrow martensitic transformation temperature (MTT) range (sometimes called intervals) brings difficulties to well control the actuating process [5]. The lack of strain-hardening on the strain-stress curve also hinders the application of NiTi alloys. In recent years, functionally graded NiTi alloys characterized by progressive transformation on loading or on cooling have been regarded as an efficient solution to resolve the problems mentioned above $[6,7]$.

Functionally graded properties can be achieved by introducing microstructural, compositional or geometrical gradients in NiTi alloys. Relevant technologies for formation of the gradients 
mentioned above actually include physical vapor deposition (PVD) [8], diffusion annealing treatment $[6,9,10]$, chemical treatment [11] and laser annealing [12-14]. The selective laser melting (SLM) method as a kind of additive manufacturing technology can be another novel way to fabricate a functionally graded NiTi alloy $[7,15]$. In our previous studies, the laser beam energy dependence of martensitic transformation in SLM fabricated NiTi alloy was observed [16]. The microstructure, crystallographic texture, functional performance vary with the SLM processing parameters $[17,18]$. Based on the obtained results, the three-dimensional functionally graded NiTi alloy is designed, fabricated and investigated in the present study.

The near equiatomic NiTi alloy powder was produced through electrode induction-melting gas atomization (EIGA) from a Ni59.68Ti40.32 (wt. \%) ingot. The chemical composition of the powder was analyzed to be Ti: $44.48 \%$, Ni: $55.98 \%$, O: $0.046 \%$, Fe: $0.004 \%$ and C: $0.0025 \%$ with methods of inductively coupled plasma-atomic emission spectroscopy (ICP-AES) and infrared method after fusion under inert gas. The phase constitution determined by XRD analysis shows solely the B2 phase for the alloying powder. A commercial SLM machine of Concept Laser (Mlab-R) was used to fabricate samples under argon atmosphere $(99.99 \%)$ with the oxygen content stabilized at the level of $300 \mathrm{ppm}$. A meander pattern with the scanning angle alternated by $67^{\circ}$ upon the precedent layer was employed to minimize the thermal stress between layers. A repetitive laser scanning strategy with the schematic illustration shown in Fig. 1 has been explored to fabricate the functionally graded NiTi alloy.

As shown in Fig. 1, the gradient direction (GD) referring to the changing direction of microstructures and functionality is set to be perpendicular to the building direction (BD). In each single powder layer, a first laser scan with constant power of $60 \mathrm{~W}$ is initially proceeded as shown in Fig. 1a, followed by another subsequent laser scan with varied laser power from $0 \mathrm{~W}$ to $95 \mathrm{~W}$ amongst different gradient areas as shown in Fig. 1b. The power increment was set to be $5 \mathrm{~W}$, hence a gradient of twenty parts with the second repetitive laser power of $0,5,10,15,20,25,30$, $35,40,45,50,55,60,65,70,75,80,85,90,95 \mathrm{~W}$ was obtained. Different gradient areas were labeled as $60 \mathrm{~W}-X \mathrm{~W}$, where the " $X$ " refers to the second repetitive laser power. Except the laser power, other scanning parameters all keep constant: layer thickness ( $t$ ) is $25 \mu \mathrm{m}$, scanning speed ( $v$ ) is $440 \mathrm{~mm} / \mathrm{s}$ and hatch space (h) is $110 \mu \mathrm{m}$. It is worth noting that the gradient width marked in Fig. $1 \mathrm{~b}$ can also be purposely adjusted. The Fig. 1c shows a cubic specimen with the size of 10 $\mathrm{mm} \times 10 \mathrm{~mm} \times 10 \mathrm{~mm}$ and gradient width of $0.5 \mathrm{~mm}$, as well as another rectangular specimen with size of $5 \mathrm{~mm} \times 8 \mathrm{~mm} \times 40 \mathrm{~mm}$ and gradient width of $2 \mathrm{~mm}$.

The samples for SEM observations were prepared by mechanical polishing followed by chemical etching with the reagent composed of $3.2 \% \mathrm{HF}, 14.1 \% \mathrm{HNO}_{3}$ and $82.7 \% \mathrm{H}_{2} \mathrm{O}$ in volume fraction. The XRD detection on the single gradient area was performed on samples which were cut individually from the graded NiTi alloy sample (gradient width of $2 \mathrm{~mm}$ ). The XRD tests were performed at ambient temperature on Rigaku D-Max 2400 with a scanning step of $0.01^{\circ}$ and a 
wavelength of $0.15406 \mathrm{~nm}(\mathrm{Cu}-\mathrm{K} \alpha 1)$. The EBSD analysis was performed on the central part of the graded tensile specimen in the FEI NanoSEM450 field-emission SEM equipped with a NordlysMax2 EBSD detector. The specimen for DSC related to the graded NiTi alloy was prepared by several steps. Firstly, a piece of thin sheet on the GD-TD plane was cut, then a needle with axial direction parallel to the GD was cut from the thin sheet. Finally, the needle was cut into small pieces with scissor and collected for DSC experiment. A Netzsch DSC 200 F3 with a heating/cooling rate of $10{ }^{\circ} \mathrm{C} / \mathrm{min}$ in nitrogen atmosphere was used to estimate the MTTs. The normalized flat tensile specimens with $5 \mathrm{~mm} \times 20 \mathrm{~mm} \times 2 \mathrm{~mm}$ gage dimensions and gradient width of $1 \mathrm{~mm}$ was directly deposited on the NiTi alloy substrate. Tensile test was conducted at room temperature with approximately $25{ }^{\circ} \mathrm{C}$. The tensile direction and transverse direction are parallel to the GD and the $\mathrm{BD}$, respectively. The cyclic tensile tests were conducted with strain increments of $0.3 \%$ and the strain rate of $10^{-3} \mathrm{~s}^{-1}$ on the INSTRON 3369 machine.

Fig. 2 displays the primary microstructural characterization on the graded NiTi alloy. It is observed from the SEM images in Fig. 2a that microstructural gradients exist on the BD-GD plane amongst the six single gradient areas $(60 \mathrm{~W}-15 \mathrm{~W}, 60 \mathrm{~W}-30 \mathrm{~W}, 60 \mathrm{~W}-45 \mathrm{~W}, 60 \mathrm{~W}-60 \mathrm{~W}, 60 \mathrm{~W}-75 \mathrm{~W}$ and $60 \mathrm{~W}-90 \mathrm{~W})$. The trace of molten pools can be clearly seen in all images. More B19' phase can be observed and its grain size seems coarsened with the increase of the second repetitive laser power. Further, small number of spherical defects was noticed, especially for the single gradient samples of $60 \mathrm{~W}-75 \mathrm{~W}$ and $60 \mathrm{~W}-90 \mathrm{~W}$. The molten pool was probably over-heated when the second repetitive scanning laser power is higher enough than $75 \mathrm{~W}$.

The EBSD maps conducted on the central part of the tensile specimen are displayed in Fig. $2 \mathrm{~b}$ and $2 \mathrm{c}$ in terms of inverse pole figures (IPFs-BD). Some black areas corresponding to high internal residual stress or defects were revealed. As the gradient width was set to be $1 \mathrm{~mm}$, the EBSD maps contain at least two gradient boundaries. Accordingly, the microstructure around the gradient boundaries is metallurgically continuous. Concerning on the texture shown by the IPFs-BD, there is no big difference between the gradient areas contained in the maps. That is because the adjacent gradient areas were fabricated with similar SLM fabrication processing parameters. Indeed, the incremental of repetitive laser power with $5 \mathrm{~W}$ was purposely designed in order to avoid sharp changes of microstructure and further guarantee the continuous change of microstructures.

Fig. $2 \mathrm{~d}$ reveals the XRD profiles corresponding to the SEM images shown in Fig. 2a. Peak separation and fitting processes were performed. Final indexations were displayed above the profiles. An example of the separated and fitted peaks for the single gradient area of $60 \mathrm{~W}-90 \mathrm{~W}$ was enlarged in Fig. 2d. It is clearly seen the martensitic B19' phase is prone to form in the sample fabricated with higher repetitive laser scanning powers. In detail, the volume fraction of B19' phase was measured to increase from $28.3 \%$ for the single gradient area of $60 \mathrm{~W}-15 \mathrm{~W}$ to $85.6 \%$ for the single gradient area of $60 \mathrm{~W}-90 \mathrm{~W}$. Such increasing volume fraction of B19' phase 
with higher repetitive laser power is in accordance with microstructure gradient. This is also consistent with the conclusion obtained in previous investigations that higher energy density leads to more volume fraction of B19' phase [15-18]. Reasons can be attributed to the evaporation of $\mathrm{Ni}$ element under higher energy density that eventually leads to the increase of MTTs [19, 20].

The DSC curves for single gradient areas and the whole graded NiTi alloy sample are displayed in Fig. 3a and Fig. 3b, respectively. The MTTs including $M_{s}, M_{f}, A_{s}$, and $A_{f}$ were all measured and listed in Table 1. In fact, each MTT increases with the second repetitive laser power. That is most probably due to evaporation of $\mathrm{Ni}$ element at higher laser power as shown by chemical composition analysis via inductively coupled plasma (ICP) method [21]. The MTT interval $\left(\Delta \mathrm{T}_{\mathrm{A} \rightarrow \mathrm{M}}=M_{s}-M_{f}\right)$ shown in the fourth columns of Table 1 actually locates between $66.6{ }^{\circ} \mathrm{C}$ and $74.1{ }^{\circ} \mathrm{C}$ for single gradient areas. But this value is as higher than $118.1{ }^{\circ} \mathrm{C}$ for the graded NiTi alloy. The $\Delta \mathrm{T}_{\mathrm{M} \rightarrow \mathrm{A}}$ values $\left(\Delta \mathrm{T}_{\mathrm{M} \rightarrow \mathrm{A}}=A_{s} / R_{s}-A_{f}\right)$ listed in the seventh columns shows similarity with the $\Delta \mathrm{T}_{\mathrm{A} \rightarrow \mathrm{M}}$. Apparently, a nearly doubled MTT interval was obtained in the graded NiTi alloy. What is also interesting to mention is some additional peaks highlighted by red arrows in Fig. 3. In contrast to the origin of the additional peaks as microstructural non-uniform or $\mathrm{R}$ phase $[15,16,19]$ in Fig. 3a for single gradient area, a more reasonable explanation for additional peaks in Fig. $3 \mathrm{~b}$ is the overlapping of MTT peaks resulting from multi-gradients. Therefore, the phase transformation gradient on cooling and heating was realized on the graded NiTi alloy.

The cyclic tensile curves consisting of strain increment of $0.3 \%$ followed by stress release until fracture are displayed in Fig. 4a. The Young's modulus, initial yielding stress, elongation and ultimate tensile stress were estimated to be approximately $32 \mathrm{GPa}, 130 \mathrm{MPa}, 6.88 \%$ and $449 \mathrm{MPa}$, respectively. It can be observed that hysteresis loops between loading and unloading paths become widened once the applied strain exceeds the first yielding point of $0.35 \%$. Additionally, the tensile curves for the functionally graded NiTi alloy reveal an exceptional strain-hardening effect of more than $300 \mathrm{MPa}$, which is unusual for NiTi alloys. These two facts on the functionality gradient are due to involvement of multiple deformation mechanisms.

In order to characterize the functionality gradient, the evolution of mechanical recoverable strain with the underlying deformation mechanisms was analyzed. As shown in Fig. 4b, the obtained mechanical recoverable strain calculated to be the difference value between the applied strain and the residual strain was plotted as the function of applied strain and highlighted by the solid black circle lines. It is surprising to find that the mechanical recoverable strain increases continuously with the applied strain. Compared to this continuous increasing relationship, the SLM fabricated NiTi alloys fabricated with non-functional gradients do behave differently as shown in the Fig. $4 \mathrm{~b}$ with open grey circle lines. Similar evolution of mechanical recoverable strain shown in other shape memory alloys with non-functional gradient can also be evidenced [22-25].

The underlying deformation mechanisms are explored hereafter to explain the continuously 
linear increase of mechanical recoverable strains. As observed from SEM images in Fig. 2a and confirmed by XRD profiles in Fig. 2d, more B19' martensite but less B2 phase were formed in the gradient areas fabricated with higher repetitive laser power. So, when the external stress imposed on the graded NiTi alloy exceeds the elastic limit, the gradient areas containing more B19' take a priority to deform via the reorientation of the pre-existing martensitic B19' twinning variants. As the reorientation process cannot be reversed after stress releasing in the present case, the loading and unloading paths are nearly overlapping at this stage $(0.5 \%<$ applied strain $<1.5 \%)$. With the increase of the applied stress, the gradient areas which contain more B2 phase with lower repetitive laser power deform subsequently via stress-induced martensitic B19' transformation. Larger hysteresis loops were hence left on the cyclic tensile curves as clearly shown in Fig. 4b. The activation of the latter deformations enables the mechanical recoverable strain to increase linearly.

The functionally graded NiTi alloys have already been successfully fabricated with various methods, like diffusion annealing [8] and laser annealing [10, 12-14]. The excellent strain-hardening highlighted by $\Delta \sigma$ in Fig. $4 \mathrm{a}$ was also found to be $300 \mathrm{MPa}$ by other reference [9]. The importance of the present study lies on two points. Firstly, the graded NiTi alloy can be fabricated with one-way SLM process without additional treatment. Further, the intrinsic feature of the additive manufacturing ensures a potentially high precision of gradient control via adjustment of the gradient width, density and fabrication strategy. Although the transformation gradient was characterized previously [6-16], the value of MTT intervals $\left(\Delta T_{A \rightarrow M}\right.$ and $\left.\Delta T_{M \rightarrow A}\right)$ which can be measured to be approx. $80 \mathrm{~K}$ [12] and $100 \mathrm{~K}$ [10] were emphasized rarely. This value reached approximately $120 \mathrm{~K}$ in the present case. As a matter of fact, the mechanical recoverable strain shown in Fig. 4b would further increase and finally stabilize at quite higher stress level where the plastic deformation would comprehensively occur. Therefore, processing parameters for the SLM needs to be further optimized.

In summary, a functionally graded NiTi alloy has been fabricated with selective laser melting method based on the design of a repetitive scanning strategy. The microstructural observations reveal a continuous microstructure evolution amongst different gradient areas and a well metallurgical fusion zone on the gradient boundaries. The microstructure graident is explicated by the increasing volume fraction of martensitic B19' phase along the gradient direction. The martensitic transformation as the origin of the functional properties is characterized by MTT intervals. The forward and backward MTT intervals of $\Delta \mathrm{T}_{\mathrm{A} \rightarrow \mathrm{M}}$ and $\Delta \mathrm{T}_{\mathrm{M} \rightarrow \mathrm{A}}$ are higher than $118^{\circ} \mathrm{C}$ and $125{ }^{\circ} \mathrm{C}$, respectively. The functionality of the graded NiTi alloy were characterized via analysis of mechanical recoverable strain and deformation mechanisms. The gradient area containing more B19' phase deforms initially via the reorientation of martensitic B19' variants. Other gradient area containing more B2 phase deforms subsequently via stress-induced martensitic transformation at higher applied stress level. The superimposed multi-deformation mechanisms lead to the continuous increase of mechanical recoverable strain and exceptional 
strain-hardening effect.

More works concerning on the in situ microstructural observation and in situ synchrotron $\mathrm{X}$-ray radiation diffraction in order to characterize more precisely the deformation details is now ongoing. A more accurate evaluation to unveil the gradient change of shape for the functionally graded NiTi is also planned. The prospects on the functionally graded NiTi alloy can be explored for biomedical and smart engineering devices as controllable self-driving actuator.

The authors acknowledge Dr. J.X. Lin in Fujian Institute of Research on the Structure of Matter, Chinese Academy of Sciences for providing facilities of additive manufacturing. This work was financially supported by the project supported by the National Science Foundation for Young Scientists of China (Grant No. 51801199).

\section{References:}

[1] K. Otsuka, X. Ren, Recent developments in the research of shape memory alloys, Intermetallics 7 (1999) 51-528.

[2] W. Huang, On the selection of shape memory alloys actuators, Materials \& Design, 23(2000) $11-19$

[3] M.H. Elahinia, M. Hashemi, M. Tabesh, S.B. Bhaduri, Manufacturing and processing of NiTi implants: A review, 57(2012)911-946

[4] M.C. Carroll, C. Somsen, G. Eggeler, Multiple-step martensitic transformation in Ni-rich NiTi shape memory alloys, Scripta Materialia, 50(2004)187-192

[5] Q.L. Meng, H. Yang, Y. Liu, T. Nam, Transformation intervals and elastic strain energies of B2-B19' martensitic transformation of NiTi, Intermetallics 18 (2010) 2431-2434.

[6] Q.L. Meng, H. Yang, Y. Liu, T. Nam, Compositionally graded NiTi plate prepared by diffusion annealing, Scripta Materialia 67 (2012) 305-308.

[7] M. Naebe, K. Shirvanimoghaddam, Functionally graded materials: A review of fabrication and properties, Applied Materials Today 5 (2016) 223-245.

[8] P.F. Rodrigues, F.M.B. Fernandes, R. Magalhaes, E. Camacho, A. Lopes, A.S. Paula, R. Basu, N. Schell, Thermo-mechanical characterization of NiTi orthodontic archwires with graded actuating forces, Journal of the Mechanical Behavior of Biomedical Materials, 107(2020)103747.

[9] B.S. Shariat, Q.L. Meng, A.S. Mahmud, Z. Wu, R. Bakhtiari, J. Zhang, F. Motazedian, H. Yang, G. Rio, T. Nam, Y. Liu, Functionally graded shape memory alloys: Design, fabrication and experimental evaluation, Materials and Design 124 (2017) 225-237.

[10] Q. Meng, Y. Liu, H. Yang, B.S. Shariat, T. Nam, Functionally graded NiTi strips prepared by laser surface anneal, Acta Materialia 60 (2012) 1658-1668.

[11] C.L. Chu, C.Y. Chung, Y.P. Pu, Graded surface structure in chemically polished NITi shape 
memory alloy after $\mathrm{NaOH}$ treatment, Scripta Materialia 52(2005)1117-1121.

[12] Q. Meng, Y. Liu, H. Yang, T. Nam, Laser annealing of functionally graded NiTi thin plate, Scripta Materialia 65(2011)1109-1112.

[13] A.S. Mahmud, Y. Liu, T. Nam, Gradient anneal of functionally graded NiTi, Smart Materials and Structures 17(2008)015031.

[14] X.Wang, Y. Bellouard, J.J. Vlassak, Laser annealing of amorphous NiTi shape memory alloy thin films to locally induce shape memory properties, Acta Materialia 53(2005)4955-4961.

[15] X.B. Wang, M. Speirs, S. Kustov, Selective laser melting produced layer-structured NiTi shape memory alloys with high damping properties and elinvar effect, Scripta Materialia 146(2018)246-250.

[16] Y. Yang, J.B. Zhan, B. Li, J.X. Lin, J.J. Gao, Z.Q. Zhang, L. Ren, P. Castany, T. Gloriant, Laser beam energy dependence of martensitic transformation in SLM fabricated NiTi shape memory alloy, Materialia 6(2019)100305.

[17] Y. Yang, J.B. Zhan, Z.Z. Sun, H.L. Wang, J.X. Lin, Y.J. Liu, L.C. Zhang, Evolution of functional properties realized by increasing laser scanning speed for the selective laser melting fabricated NiTi alloy, Journal of Alloys and Compounds, 804(2019)220-229

[18] Z.X. Khoo, J. An, C.K. Chua, Y.F. Shen, C.N. Kuo, Y. Liu, Effect of heat treatment on repetitively scanned SLM NiTi shape memory alloy, Materials (Basel) 12(2019)77.

[19] S. Saedi, N.S. Moghaddam, A. Amerinatanzi, M. Elahinia, H.E. Karaca, On the effects of selective laser melting process parameters on microstructure and thermomechanical response of Ni-rich NiTi, Acta Materialia 144(2018)552-560.

[20] Z. Zeng, B.Q. Cong, J.P. Oliveira, W.C. Ke, N. Schell, B. Peng, Z.W. Qi, F.G. Ge, S.S. Ao, Wire and arc additive manufacturing of a Ni-rich NiTi shape memory alloy: Microstructure and mechanical properties, Additive manufacturing 32(2020)101051.

[21] M. Speirs, X. Wang, S.V. Baelen, A. Ahadi, S. Dadbakhsh, On the transformation behavior of the NiTi shape-memory alloy produced by SLM, Shape Memory and Superelasticity 2(2016)310-316.

[22] Y. Yang, P. Castany, E. Bertrand, M. Cornen, J.X. Lin, T. Gloriant, Stress release-induced interfacial twin boundary $\omega$ phase formation in a $\beta$ type Ti-based single crystal displaying stress-induced $\alpha "$ martensitic transformation, Acta Materialia, 149(2018)97-107.

[23] M. Besse, P. Castany, T. Gloriant, Mechanisms of deformation in gum metal TNTZ-O and TNTZ titanium alloys: a comparative study on the oxygen influence, Acta Materialia, 59(2011)5982-5988.

[24] L. Héraud, P Castany, D. Laillé, T. Gloriant, In Situ synchrotron X-ray diffraction of the martensitic transformation in superelastic $\mathrm{Ti}-27 \mathrm{Nb}$ and NiTi Alloys: a comparative study, Materials today: proceedings 2(2015)917-920.

[25] K. Otsuka, X. Ren, Physical metallurgy of Ti-Ni-based shape memory alloys, Progress in Materials Science 50(2005) 511-678. 
Table 1: Characteristic martensitic transformation temperatures (MTTs) measured from several selected gradient areas and from the whole graded NiTi alloys.

\begin{tabular}{ccccccc}
\hline Specimens & $\begin{array}{c}M_{s} \\
{ }^{\circ} \mathrm{C}\end{array}$ & $\begin{array}{c}M_{f} \\
{ }^{\circ} \mathrm{C}\end{array}$ & $\begin{array}{c}\Delta \mathrm{T}_{\mathrm{A} \rightarrow \mathrm{M}}, \\
{ }^{\circ} \mathrm{C}\end{array}$ & $\begin{array}{c}A_{s} \text { or } R_{s} \\
{ }^{\circ} \mathrm{C}\end{array}$ & $\begin{array}{c}A_{f} \\
{ }^{\circ} \mathrm{C}\end{array}$ & $\begin{array}{c}\Delta \mathrm{T}_{\mathrm{M} \rightarrow \mathrm{A},} \\
{ }^{\circ} \mathrm{C}\end{array}$ \\
\hline $60 \mathrm{~W}-15 \mathrm{~W}$ & 14.5 & -52.1 & 66.6 & -19.6 & 28.3 & 47.9 \\
\hline $60 \mathrm{~W}-30 \mathrm{~W}$ & 20.5 & -46.2 & 66.7 & -14.2 & 50.6 & 64.8 \\
\hline $60 \mathrm{~W}-45 \mathrm{~W}$ & 28.3 & -39.8 & 68.1 & -8.8 & 51.8 & 60.6 \\
\hline $60 \mathrm{~W}-60 \mathrm{~W}$ & 38.7 & -33.7 & 72.4 & 50.6 & 88.9 & 38.3 \\
\hline $60 \mathrm{~W}-75 \mathrm{~W}$ & 42.5 & -30.2 & 72.7 & 56.9 & 99.1 & 42.2 \\
\hline $60 \mathrm{~W}-90 \mathrm{~W}$ & 46.4 & -27.7 & 74.1 & 67.8 & 105.4 & 37.6 \\
\hline Graded NiTi & $\mathbf{6 8 . 1}$ & $<-\mathbf{5 0}$ & $>\mathbf{1 1 8 . 1}$ & $\mathbf{- 1 1 . 9}$ & $\mathbf{1 1 3 . 7}$ & $\mathbf{1 2 5 . 6}$ \\
\hline
\end{tabular}


(a) The first laser scan $(60 \mathrm{~W})$

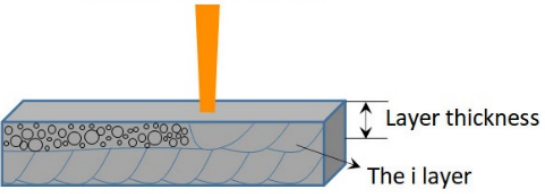

(b) - The repetitive laser scan

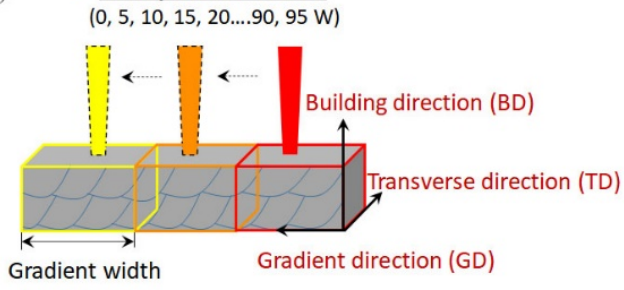

(c)

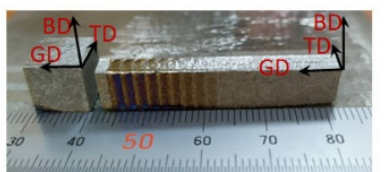

Fig. 1 Schematic illustration for the functionally graded NiTi alloy: (a) the first laser scan with a constant laser power of $60 \mathrm{~W},(\mathrm{~b})$ the repetitive laser scan with the varied laser power from $5 \mathrm{~W}$ to $95 \mathrm{~W},(\mathrm{c})$ two typical graded NiTi specimens.

(a)
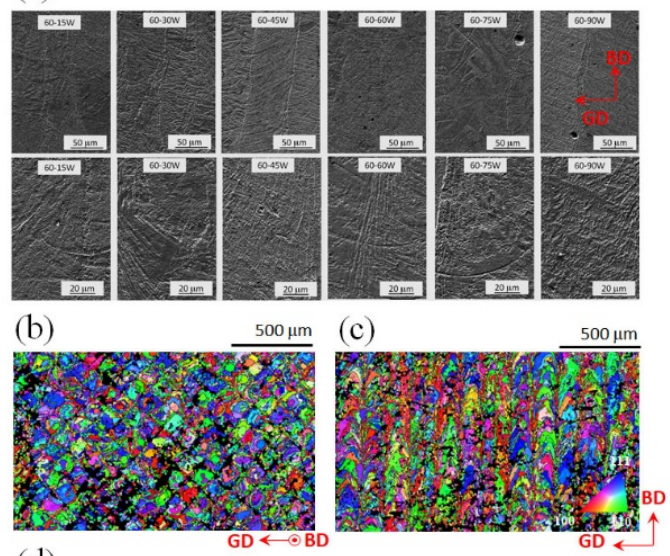

(d)

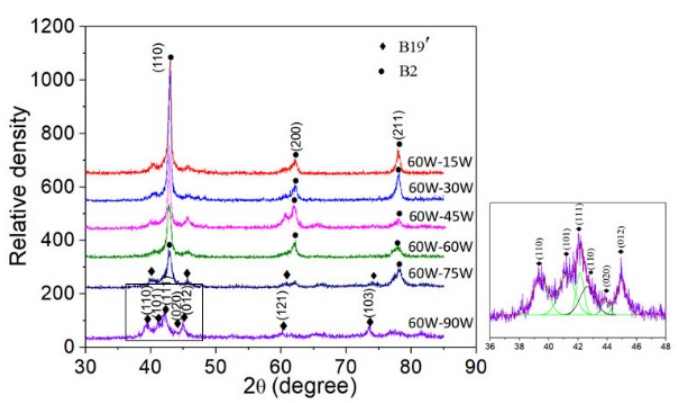

Fig. 2 Primary characterization for the functionally graded NiTi alloy: (a) the SEM microstructures of several selected gradient areas, (b, c) the IPFs mapping observed from different planes of SLMed sample, (d) the XRD profiles of several selected gradient areas. 
(a)

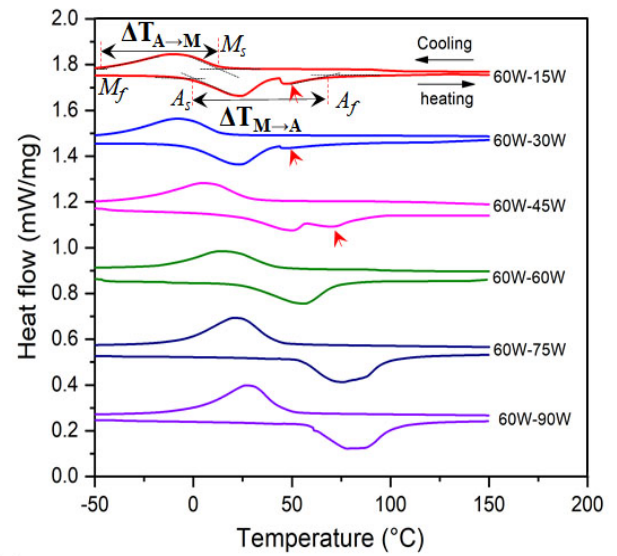

(b)

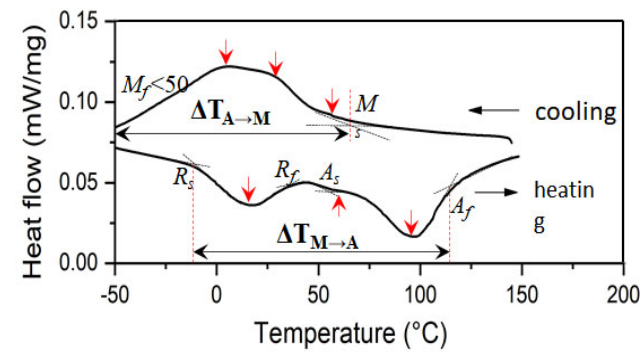

Fig. 3 (a) DSC curves for (a) different single gradient samples and (b) the whole functionally graded NiTi alloy

(a)

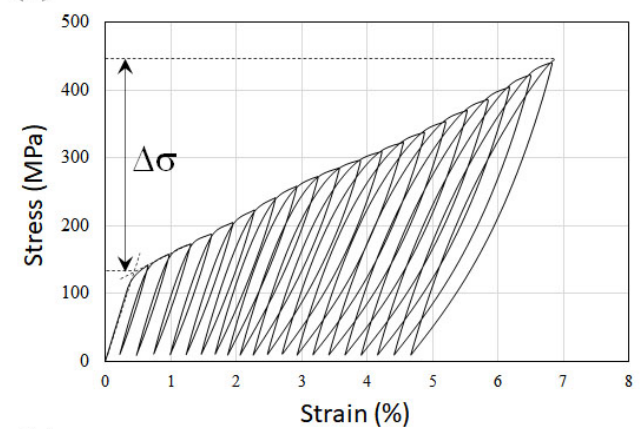

(b)

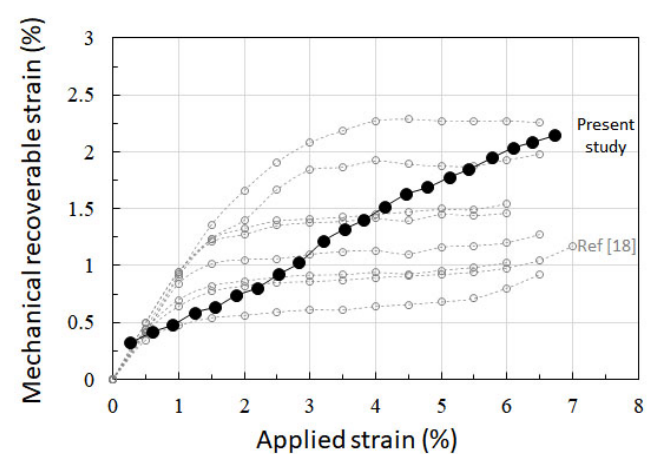

Fig. 4 (a) Cyclic tensile curve for the functionally graded NiTi alloy and (b) the relationship between the mechanical recoverable strain and applied strain compared with SLM fabricated NiTi alloy without functional gradient 